\title{
Apprendre à lire et idéologie politique
}

L'alphabet républicain du citoyen Chemin

\section{Jean-Gérard Lapacherie}

\section{(2) OpenEdition \\ Journals}

Édition électronique

URL : http://journals.openedition.org/trema/1661

DOI : $10.4000 /$ trema. 1661

ISSN : 2107-0997

Éditeur

Faculté d'Éducation de l'université de Montpellier

Édition imprimée

Date de publication : 1 juillet 2000

Pagination : 27-41

ISSN : 1167-315X

Référence électronique

Jean-Gérard Lapacherie, «Apprendre à lire et idéologie politique », Tréma [En ligne], 17 | 2000, mis en ligne le 01 juillet 2000, consulté le 06 mai 2019. URL : http://journals.openedition.org/trema/1661 ; DOI : 10.4000/trema.1661

Ce document a été généré automatiquement le 6 mai 2019.

Trema 


\title{
Apprendre à lire et idéologie politique
}

\author{
L'alphabet républicain du citoyen Chemin
}

\author{
Jean-Gérard Lapacherie
}

En l'an II de la République (qui s'étend du 21 septembre 1793 au 20 septembre 1794), le citoyen Chemin, imprimeur et libraire de son état, publie

un Alphabet républicain, ${ }^{1}$ dont la page de titre précise qu'il est «orné de gravures et suivi de conversations à la portée des enfants, propres à leur inspirer l'amour de la liberté, de l'égalité et de toutes les

vertus républicaines; et à les mettre en état de bien entendre la déclaration des droits et la constitution ». Il se présente matériellement comme un livret de petit format $(10,5 \mathrm{~cm} \times 15 \mathrm{~cm})$ qui tient dans la

main. Il comprend 38 pages de texte et il est précédé de 9 pages de gravures et suivi de 6 pages

d'un avis contenant la liste des publications de Chemin fils.

\section{Un abécédaire}

1 Alphabet a le sens d'abécédaire. Dans son Dictionnaire de la Langue française, Emile Littré définit, entre autres acceptions, l'alphabet comme «un petit livre qui contient les lettres de l'alphabet et les éléments de la lecture ». Ce livret est destiné à apprendre à lire, et cela se fait, ou est censé se faire, par la connaissance du seul alphabet. Autrement dit, selon Chemin, pour lire un texte, il suffit de connaître ou de reconnaître des lettres et, en les proférant à haute voix, de les combiner pour former des syllabes. Il s'agit là d'une conception particulière de la lecture, et aussi de l'écriture, dont, dans cet article, je me propose, entre autres objectifs, d'isoler les présupposés. De plus, l'adjectif républicain, qui qualifie alphabet, a un sens très fort, qui renvoie à l'idéologie des Conventionnels. 
Chemin est robespierriste. Son Alphabet [...] se termine par une invocation à l'Etre Suprême. Il est lié au nouveau et tout jeune régime, créé le 20 septembre 1792, qui a ouvert (ou voulu ouvrir) une nouvelle ère, avec son comput (An II) ; son calendrier, ses nouvelles divisions, etc. Il opère une rupture, à la fois profonde et violente, avec plusieurs siècles d'histoire. C'est la fin de la monarchie, certes, mais aussi celle d'une religion « officielle». En effet, le véritable antonyme de républicain est non pas monarchique mais latin, au double sens de relatif à la Rome antique, dont la langue était le latin, et de « catholique » ou de «chrétien ». Dans le titre, des relations sont explicitement établies entre la lecture et l'organisation de l'Etat, entre une méthode d'apprentissage de la lecture et une idéologie politique. Ces relations vont dans une seule et même direction, qui fait de la lecture le véhicule de valeurs à diffuser ou à imposer aux enfants. Cet état de choses n'est pas propre à la Révolution. Il ne fait que continuer une situation antérieure, puisque, dans les petites écoles d'Ancien Régime, étaient enseignées trois matières: religion, morale et lecture.

2 Ce sont les rapports qui existent dans cet Alphabet républicain entre la lecture et l'idéologie que je vais saisir dans cet article. Pour cela, dans un premier temps, j'étudierai le contexte politique et historique dans lequel s'inscrit cet Alphabet [...]; puis je montrerai qu'à aucun moment, Chemin ne fonde l'apprentissage des signes de l'alphabet et en conséquence celle de la lecture sur une analyse des signes, de quelque nature qu'elle fût, phonétique, linguistique ou autre ; enfin, je montrerai que le caractère républicain que Chemin attribue à sa méthode (de façon indue, à mon sens, puisqu'elle est pure propagande) n'a rien en commun avec les principes qu'établit au même moment Condorcet dans ses Mémoires sur l'instruction publique et qui fondent ce que certains philosophes ou penseurs contemporains, ${ }^{2}$ tels que Alain Finkielkraut, Jacques Muglioni, ${ }^{3}$ Catherine Kintzler, Régis Debray, nomment « l'école de la République ».

\section{Un auteur « engagé »}

3 De Chemin fils, dit Chemin-Dupontès, prénommé Jean-Baptiste, ll' $^{2}$ histoire a retenu qu'il est né en 1760 et mort vers 1852, qu'il s'est établi comme imprimeur libraire dans l'île de la Cité en 1789, qu'il a adhéré aux idées révolutionnaires " avec modération », et que sa principale activité pendant dix ans a consisté à imprimer ses propres livres, dont $\mathrm{l}^{\prime}$ Alphabet républicain, plusieurs fois réédité, ainsi que L'Ami des jeunes patriotes ou Catéchisme républicain, "ouvrage (qui) contient les actions héroïques des jeunes martyrs de la liberté, les principes de la lecture, de l'écriture et du calcul, l'histoire abrégée de la révolution depuis 1789, la déclaration des droits, la constitution, l'instruction sur l'année républicaine et sur les saisons avec les meilleurs hymnes civiques"; la Constitution française, précédée de la déclaration des droits de l'homme et du citoyen ; la Déclaration des droits de l'homme et du citoyen, etc. D'après ses biographes, Chemin était « un homme paisible, éminemment moral et bon disciple de Rousseau (qui a créé) de toutes pièces la théoanthropophilie, culte familial, déiste et humanitaire, dont il publia le Manuel». Plus tard, il a adhéré à la franc-maçonnerie et semble avoir abandonné l'imprimerie pour devenir maître de pension, puisqu'en 1824, il est considéré comme "ancien instituteur ». En bref c'est un idéologue déiste, aimant l'humanité, lecteur de Rousseau, qui édite, imprime et vend les livres qu'il a écrits.

4 L'Alphabet républicain doit être aussi replacé dans le contexte troublé de l'année 1794. Des hommes politiques alors ont des préoccupations en matière de lecture, dont se font écho les députés Barrère et l'abbé Grégoire. ${ }^{5}$ Les Français peuvent-ils respecter les droits 
de l'homme et en jouir, s'ils ne sont qu'une minorité à entendre la langue dans laquelle sont rédigées la Déclaration des droits de l'homme, la Constitution, les lois nouvelles ? Les Français peuvent ils connaitre leurs droits si la majorité d'entre eux reste analphabète? Aussi fait-on traduire la déclaration, la constitution de 1791, les décrets et les textes de loi dans les langues régionales; aussi nomme-t-on des instituteurs publics dans les communes frontalières ou proches des frontières qui sont chargés d'apprendre à lire, à écrire et à compter aux enfants, et aussi de lire en public le dixième jour de chaque décade les textes et décrets, et de les commenter. Chemin, en commerçant avisé, sent que la situation est commercialement favorable à son entreprise d'imprimeur. En mettant son Alphabet [...] sur le marché, il entend certes " mettre (les enfants) en état de bien entendre la Déclaration des droits, et la Constitution ", mais aussi répondre à une demande.

De plus, en décembre 1793, le cadre législatif qui régit l'enseignement de l'époque est modifié par un décret oublié, méconnu, qui porte le nom de son auteur, Gabriel Bouquier (1750-1811), écrivain, élu de la Dordogne à la Convention, membre du comité d'instruction publique qui a rédigé un rapport, transformé le 19 décembre 1793 en décret, dont l'objet est " d'organiser l'instruction publique ». Il aurait fallu écrire ré-organiser, car, à la fin de l'année 1793, l'enseignement en France est désorganisé, à cause des événements politiques, de la guerre aux frontières et surtout à la suite de l'émigration d'une partie des prêtres qui étaient les principaux (parfois les seuls) maîtres d'école, et des nobles, qui fournissaient aux petites écoles et aux collèges une bonne part de leurs élèves. Ce décret signé Bouquier, jacobin et robespierriste, pose deux principes :

- l'enseignement est libre ;

- tout citoyen (et citoyenne) peut ouvrir une école.

Dans le cadre politique français, libre signifie « indépendant de l'Etat ». Autrement dit, au début de l'An II, la République affirme solennellement par ce décret qu'il ne lui incombe pas de créer des écoles, de les faire fonctionner, de former les maîtres, mais qu'elle délègue cette responsabilité aux citoyens et qu'elle la confie aux seuls citoyens. La thèse de la liberté de l'enseignement, qui est depuis 1810 et jusqu'à aujourd'hui, celle des « cléricaux » ou de l'Eglise, celle qui a inspiré le comte de Falloux quand il fait voter, en 1850, la loi qui porte son nom, celle qui est revendiquée par les partisans de l'école confessionnelle (et/ou catholique, et/ou privée, et/ou libre), est posée, affirmée, imposée à l'origine par un régime jacobin. La liberté qui est donnée aux citoyens, pourtant, n'est pas totale. Des limites sont fixées. Les citoyens désireux d'enseigner doivent en informer la commune, déclarer les matières qui feront l'objet de leur enseignement, ne pas attenter aux bonnes mœurs et accepter la surveillance des autres citoyens.

7 Outre ces deux principes, le décret Bouquier définit ce qui est nommé le « premier degré d'instruction ». En voici les articles I et II.

«Art. I La Convention nationale charge son comité d'instruction de lui présenter les livres élémentaires des connaissances absolument nécessaires pour former des citoyens et déclare que les premiers de ces livres sont les Droits de l'homme, la Constitution, le Tableau des actions héroïques et vertueuses.

" Art. I Les citoyens et citoyennes qui se borneront à enseigner à lire, à écrire et les premières règles de l'arithmétique, seront tenus de se conformer, dans leurs enseignements, aux livres élémentaires adoptés et publiés à cet effet par la représentation nationale $»{ }^{6}$

8 La publication de l'Alphabet républicain s'inscrit dans ce cadre législatif. Dans la page de titre, Chemin précise que son abécédaire a été « accepté par la Convention nationale » et qu'il a été «approuvé par la Commune de Paris». La liste des publications de Chemin, annexée 
dans les six pages de l'avis final, confirme cela. Citons la publication $\mathrm{N}^{\circ} 5 \mathrm{~L}^{\prime} \mathrm{ami}$ des jeunes patriotes ou Catéchisme républicain; $\mathrm{N}^{\circ} 9$ Constitution française, précédée de la déclaration des droits de l'homme et du citoyen; $\mathrm{N}^{\circ} 10$ : Déclaration des droits de l'homme et du citoyen.

\section{Un bréviaire illustré}

9 Cet Alphabet, de petit format (10,5 cm de large sur $15 \mathrm{~cm}$ de haut), qui tient dans la main, comme un bréviaire, est donc destiné aux citoyens qui, dans le cadre du décret Bouquier, veulent ouvrir une école ; et même, éventuellement, à ceux qui veulent apprendre à lire et qui, pour de nombreuses raisons, ne peuvent pas fréquenter une école. Pour Chemin, la notion de classe à laquelle nous sommes familiers et qui impose que les enfants soient répartis en groupes homogènes en fonction de leur âge et de leur niveau, n'existe pas. Dans les dix conversations, un maître s'adresse à un seul destinataire, qu'il apostrophe, le nommant paternellement "mon enfant», le désignant encore par «toi» ou à qui il dit «tu ». La gravure qui illustre la lettre E montre un enfant, âgé de 8 à 10 ans, coiffé d'un chapeau orné d'une cocarde tricolore et correctement habillé (il porte des souliers) et qui joue au cerf-volant. C'est sans doute la représentation d'un des destinataires éventuels de cet Alphabet. La notion de « classe » (d'âge et de niveau) est récente; elle s'impose dans le courant du XIX ${ }^{e}$ s. Dans les petites écoles d'Ancien Régime, le maître enseignait à un groupe d'enfants âgés de 6 à 13 ans ou plus, qui suivaient le même programme, suivant une méthode individualisée. Le maître s'occupait des enfants les uns après les autres; il faisait réciter une leçon à un enfant, tandis que les autres jouaient, rêvaient ou étudiaient dans leur coin.

C'est aussi un abécédaire illustré, orné de gravures, qui ouvrent le livret et précèdent le texte, dont elles sont séparées. Les gravures sont délimitées par des filets rectangulaires. Elles contiennent une lettre imprimée en grandes capitales et en italiques, une image et le nom écrit en italiques, qui sert aussi de légende à cette image, nom qui commence par la lettre en capitales et en italiques. En Occident, les abécédaires ou les syllabaires sont des livres illustrés. Cette tradition se poursuit encore. Elle trouve sans doute ses origines dans les manuscrits et dans les pratiques d'enluminure des lettres signalant un début de chapitre. 
Figure 1

Page de titre de l' « Alphabet républicain du citoyen [...]. Paris, Éds. : Chemin Fils, 1793 ».

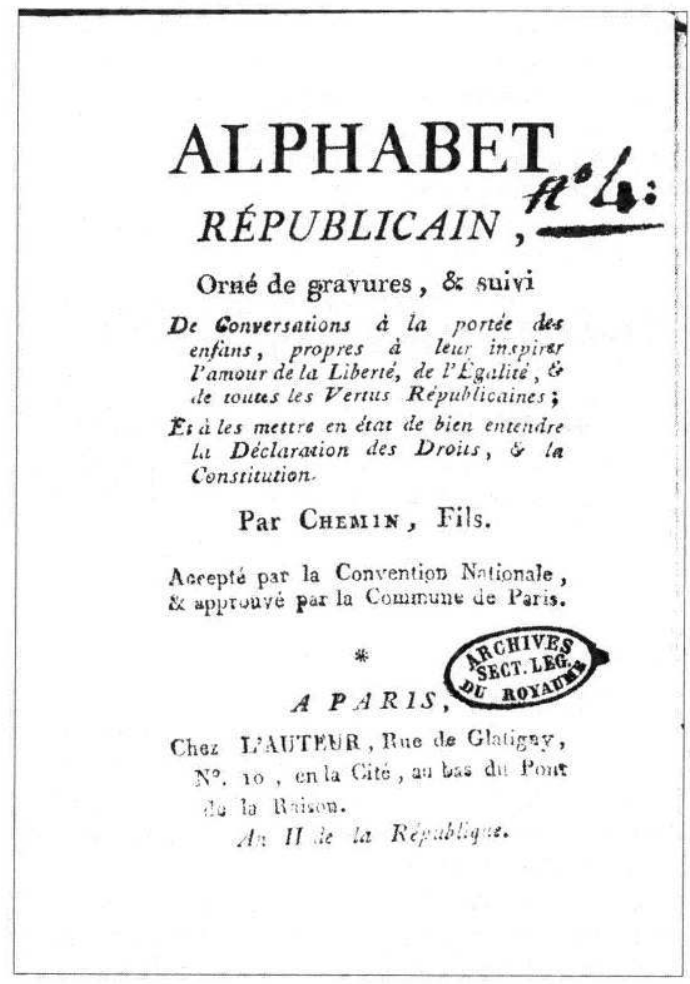

11 Une question reste en suspens : des images sont insérées dans des abécédaires ? Pourquoi les abécédaires sont-ils toujours illustrés? Certes, il n'est pas aisé de se dégager des traditions. Mais la véritable raison est ailleurs. Longtemps, notre culture s'est fait une conception réductrice de l'image. Pour elle, l'image est naturelle. Elle montre les choses telles qu'elles sont, elle reproduit le monde, elle se lit sans difficulté, au premier coup d'œil. Il s'agit d'un langage non élaboré, primitif, immédiatement interprétable, et qui, en conséquence, est censé convenir naturellement et parfaitement aux illettrés et aux enfants, que l'on renvoie souvent à la nature et que l'on identifie parfois aux primitifs. Ce n'est qu'une pure illusion, bien sûr, et idéologique.

En effet, les gravures qui ornent l'Alphabet [...] de Chemin ont un sens problématique. Sans les légendes qui les accompagnent, elles resteraient, même pour les adultes, difficiles à interpréter, ne serait-ce que parce qu'il n'est pas possible de reconnaître dans une image sans légende l'objet qui est représenté.

\section{Catalogue de signifiants graphiques}

Cet Alphabet est avant tout un catalogue, dans lequel l'auteur dresse un inventaire de signifiants graphiques, c'est-à-dire de dessins, qu'il s'agit d'identifier et de faire reconnaître aux enfants. Chemin recense tous ces signifiants : les lettres minuscules et majuscules, les lettres liées ensemble, les signes de ponctuation, les accents, les chiffres arabes et romains. La question de la valeur phonétique de ces signes n'est pas posée, ni abordée. Nulle part, Chemin ne précise à quelles unités de la langue renvoient ces signes. Ainsi, la lettre $\mathbf{E}$ est illustrée par le mot enfant : or, le premier phonème qui compose ce 
mot n'est pas un e mais un a nasal. Autre exemple : i est illustré par le mot imprimeur, dont l'initiale ne représente pas un $\mathbf{i}$, mais par un è ouvert nasal; o par oiseau, dont le signifiant phonique commence par la semi-consonne w suivie de $\mathbf{a}$. La seule analyse que l'on puisse qualifier de «linguistique » est la répartition des lettres en voyelles et en consonnes, termes qui, dans le vocabulaire grammatical du XVIII ${ }^{e}$ s., désignent des lettres, et non des sons comme aujourd'hui. Pour désigner les sons, on employait les termes de voix (pour voyelles) et d'articulations (pour consonnes).

Chemin distingue 5 voyelles et 19 consonnes, mais la lettre y qui apparait dans la liste des lettres ne figure ni dans les voyelles, ni dans les consonnes.

Cette absence de réflexion linguistique sur les relations entre les signes et ce pour quoi ils valent caractérise aussi la liste qu'il établit des syllabes. Chemin ne retient que deux combinaisons possibles. Ou bien, une consonne est suivie d'une voyelle, $\mathrm{CV}$ : exemples, ba, bi ; ou bien, une consonne est suivie d'une autre consonne et suivie d'une voyelle, CCV : exemples, bra, bri. Les autres combinaisons, très diverses en français $(\mathrm{V}, \mathrm{CVC}$, CCVC, VC, VCC, CCVCC, etc.) sont oubliées. De plus, il incombe au maitre d'apprendre toutes les combinaisons théoriquement possibles, à partir des deux seuls schémas $\mathrm{CV}$ et CCV, même si elles sont très rares en français ou même si elles n'existent pas : ainsi, quu, guu, tlu, tli, etc. Pour Chemin, l'alphabet est un catalogue de signifiants graphiques, qu'il convient de connaître et d'apprendre à combiner pour savoir lire.

Ces signifiants graphiques ne sont pas ceux de l'écriture manuscrite, uniquement les caractères de plomb qui se trouvent dans les casses d'imprimeurs. L'apprentissage qu'il propose de la lecture est autant une initiation aux signes des casses qu'un apprentissage de la lecture : la distinction entre les bas de casse et les capitales, dont le dessin varie parfois dans d'importantes proportions (a et $\mathbf{A}$ ); les lettres liées ensemble (\& ou esperluette, $æ, \propto$, ss doubles au dessin ancien, proche de celui de f, st, si, ssi, ff, fl, ffi) ; les signes de ponctuation (virgule, point virgule, deux-points, point interrogant et point admiratif). Leur emploi n'est pas enseigné. On apprend à les reconnaître, pas à les utiliser. Le savoir transmis est passif.

\section{Le modèle de l'imprimerie}

16 Chemin enseigne à lire des imprimés, non des manuscrits. Il est lui-même imprimeur, et il partage l'idéologie dominante de la fin du XVIII ${ }^{e}$ s. qui veut que l'imprimerie diffuse les lumières, émancipe, enseigne la vérité. Les textes imprimés à la lecture desquels sont préparés les enfants sont essentiellement les décrets du pouvoir politique, les lois, la constitution, la déclaration des droits de l'homme et du citoyen. La gravure intitulée " imprimerie » montre un ouvrier qui tient dans les mains deux balles avec lesquelles il enduit d'encre des caractères, avant de presser dessus une feuille. A côté, sèche une affiche sur laquelle est écrit en grosses lettres le nom décret. 
Figures 2,3 et 4 ci-dessous

Diverses pages extraites de l' " Alphabet républicain du citoyen, [...]. Paris, Éds. : Chemin Fils, 1793 ».

3

a b c def g hijk l m no

p q $\mathrm{q}$ t u v $\times$ y z

A B C D E F G H I J K I

$M$ N O P Q R S T U V X Y Z

Lettres liées ensemble:

\& If it fi fir ff fl fi ffi $a$

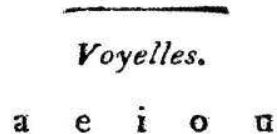

Les autrcs lettres de l'alphabet sont des Consonnes.

b c d f g h j k $1 \mathrm{~m} \mathrm{n} \mathrm{p} \mathrm{q}$

$s \mathrm{t} \times \mathrm{z}$

A)
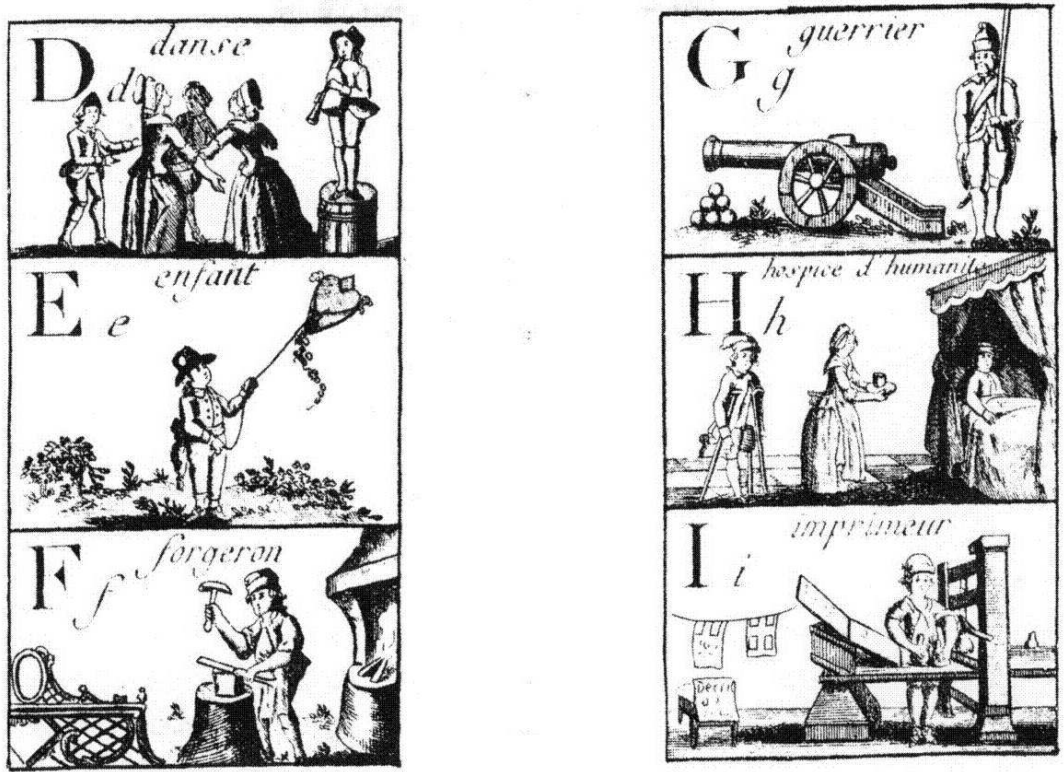


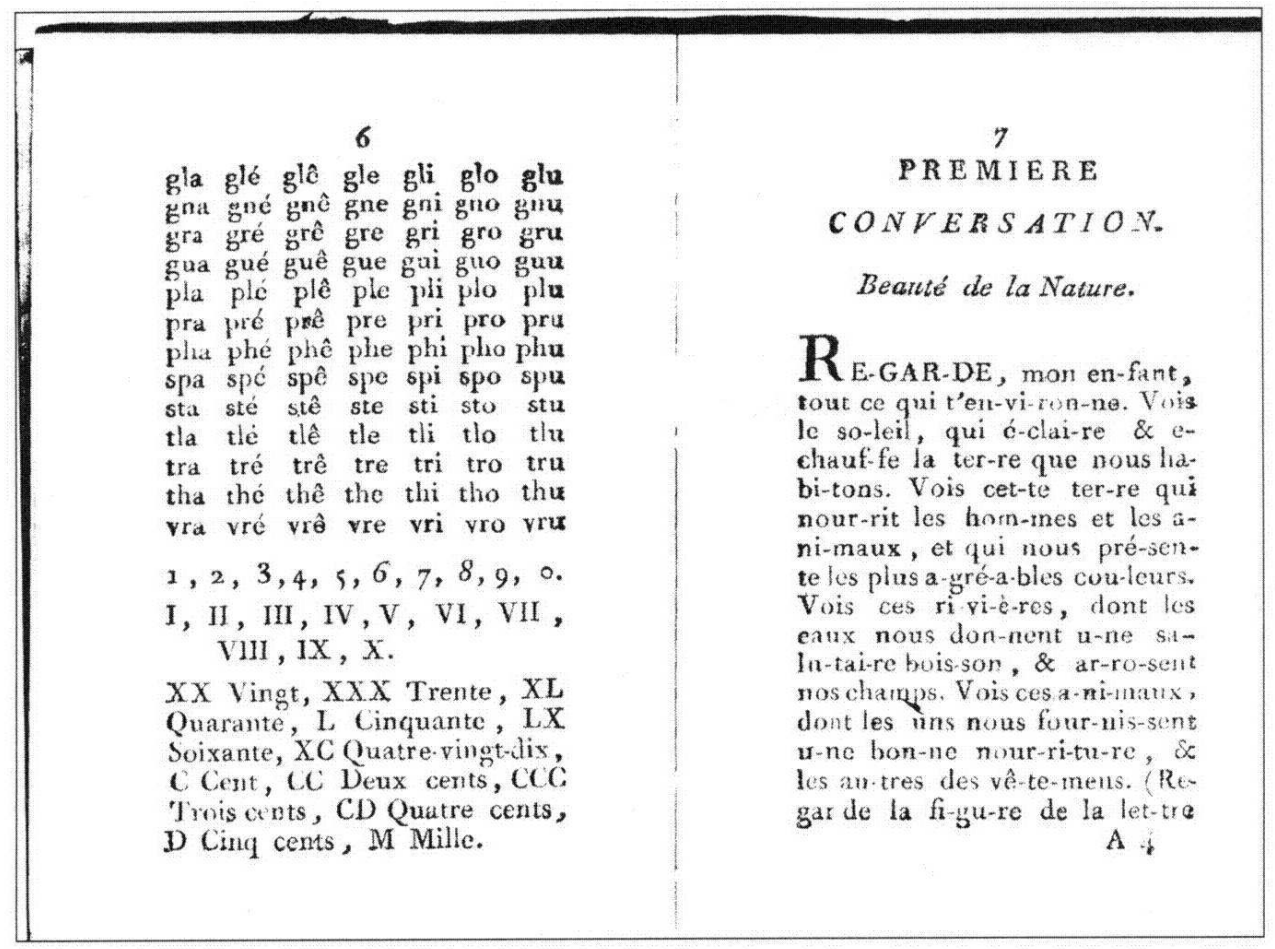

Dans les petites écoles d'Ancien Régime, étaient enseignées la religion, la morale, la lecture; et les textes qui étaient lus à l'école étaient surtout des morceaux pieux. L'enseignement de l'écriture a été longtemps réservé aux seuls maîtres écrivains, à la suite d'un privilège qui leur a été accordé en 1559. Cette dissociation entre la lecture et l'écriture a duré pendant tout le XIX ${ }^{e}$ s., sinon dans la réalité, du moins dans les lois scolaires. Dans le décret Bouquier du 19 décembre 1793, l'article II précise que les buts assignés à l'école élémentaire sont "enseigner à lire, à écrire, \& les règles de l'arithmétique ". Les deux verbes lire et écrire sont juxtaposés et séparés par une virgule. Il en va de même dans les décrets impériaux de 1806 et 1808 , où l'article 5, $6^{\mathrm{e}}$ alinéa, est formulé ainsi : «les petites écoles, où l'on apprend à lire, à écrire, et les premières notions de calcul». Dans la loi Guizot de 1833, la lecture et l'écriture sont deux matières distinctes: "L'instruction primaire élémentaire comprend nécessairement l'instruction morale et religieuse, la lecture, l'écriture, les éléments de la langue française et du calcul, le système légal des poids et mesures ". La loi Falloux du 15 mars 1850 reprend dans son article 17 l'article 1 de la loi Guizot. Il faut attendre 1882, et la loi Ferry, pour que soient associées la lecture et l'écriture. Jules Ferry, énumérant dans l'article premier les matières enseignées à l'école primaire, écrit "la lecture et l'écriture " : les deux termes sont coordonnés par la conjonction et. Votée en 1975, la loi Haby, qui énumère ainsi les matières " expression orale et écrite ; lecture; calcul ", dissocie à nouveau les deux apprentissages. ${ }^{7}$

De ce point de vue, Chemin est fidèle aux préjugés de son temps et à ceux de la tradition scolaire.

\section{Ecouter une voix qui assène un message}

Pour Chemin, que signifie lire? A l'entrée lecture, Littré, dans le Dictionnaire de la langue française, relève les quatre acceptions que voici : 
"1 $1^{e}$.) Action de lire et particulièrement, action d'une personne qui lit à haute voix.

2e.) L'action, l'habitude de lire seul et des yeux, pour son instruction ou pour son plaisir.

$3^{e}$.) La chose lue.

4e.) Instruction qui résulte de la lecture.»

Aujourd'hui, les sens que nous donnons à lecture correspondent aux acceptions 2 et 3 de ce Dictionnaire [...]. Ceux que Chemin retient sont plutôt les 1 et 4 . La lecture, pour lui, se fait à haute voix. Les listes alphabétiques sont suivies de dix textes, intitulés " conversations ", et qui sont destinés à être proférés. Dans la première conversation, les syllabes sont séparées par un tiret, ce qui suppose une lecture à haute voix. Le découpage syllabique est purement graphique, inadapté à une lecture fluide et courante. De plus, pour Chemin, la lecture est toujours très étroitement liée à la religion et à la morale. Dans les dix « conversations ", une voix adulte parle et donne des leçons de morale à un enfant. Est mise en scène une situation d'oral fictive. Conformément aux manuels en usage alors, le lecteur se trouve dans une situation passive. C'est un récepteur et, je dirais même, un réceptacle du sens. Il ne lit pas pour son plaisir mais pour être « instruit » ou recevoir des leçons que lui assène le maître. En théorie, une conversation est un échange verbal, qui suppose au moins deux locuteurs. Or, dans ces conversations, il n'y en a qu'un : la voix adulte, qui s'exprime moins qu'elle n'enjoint, essentiellement à l'impératif. Ainsi, page 27, elle ordonne : «fais tout ce qu'on te demande ». Les sujets des dix « conversations » révèlent ce qu'est la lecture pour Chemin. Les voici dans l'ordre dans lequel elles se succèdent: "Dieu tu adoreras (la religion consiste à suivre les lois de Dieu); père et mère tu honoreras (amour paternel et maternel) ; la terre tu cultiveras; à ton gouvernement tu obéiras; ta patrie tu défendras; un métier tu exerceras; un enfant sage tu seras; un homme mûr tu deviendras; heureux dans ta vieillesse tu seras." La lecture consiste en fait à proférer dix commandements qui forment la nouvelle table de lois et qui ne sont guère différents de ceux que celui que la Bible appelle l'Eternel aurait dictés à Moïse.

\section{Présupposés idéologiques}

Des présupposés idéologiques sous-tendent aussi la conception que Chemin se fait de l'enfance.

Les auteurs de dictionnaires conviennent de définir l'enfance comme une «période de la vie de tout être humain ». Une période a un début et une fin. C'est, exprimé en d'autres termes, le lieu commun des âges de la vie, grâce auquel l'enfance a été définie de l'Antiquité au XVIII ${ }^{e}$ siècle. Bien que les termes âges de la vie soient évités, c'est bien de cela qu'il s'agit. La vie humaine est divisée en âges, dits périodes, trois, quatre, cinq, ou plus. Le problème, ce sont les limites que l'on fixe à chaque âge, en particulier à l'enfance. Quand commence-t-elle et quand finit-elle? Il existe un consensus pour déterminer le début. C'est "de la naissance ", dans le Petit Larousse Illustré ; "depuis la naissance», selon Littré et Quillet, dans le Grand Robert, le Grand Larousse de la Langue Française, l' Encyclopédie Universalis; les "premières années de la vie» dans le Trésor de la Langue Française. En revanche, comme dans les dictionnaires anciens, le moment où s'achève l'enfance n'est pas établi avec précision. Littré y fixe deux fins : "vers la septième année " ou "jusqu'à treize ou quatorze ans", reprenant deux des limites proposées par les lexicographes de l'âge classique. Dans le Petit Larousse Illustré, en 1992, la limite supérieure est la puberté. Il en va de même dans le Dictionnaire Quillet de la Langue Française. La période qui précède ces changements physiologiques définit l'enfance. D'autres limites sont proposées : «l'adolescence» dans le Trésor de la Langue Française, le 
Grand Larousse de la Langue Française, le Grand Robert de la Langue Française. Dans le Dictionnaire Général de la Langue Française de Darmesteter et Hatzfel, c'est «la première période de la vie de l'homme, celle pendant laquelle son corps et son âme ne sont pas développés". Pour les auteurs de l'article «enfance » de l'Encyclopédie Universalis, l'adolescence, en revanche, est incluse dans l'enfance. La limite supérieure varie dans des proportions importantes, de sept ans à la fin de l'adolescence.

21 La période elle-même, à son tour, est divisée en sous-périodes : enfance et petite enfance ou bas âge. L'Universalis distingue les cinq sous-âges que voici :

- La première enfance ;

- L'enfant de un à trois ans, l'âge de la déambulation et du langage ;

- La période préscolaire : de trois à six ans ;

- L'âge scolaire : de six ans à l'âge de la puberté ;

- L'adolescence.

La définition du Dictionnaire Général de la Langue Française : "période pendant laquelle son corps et son âme ne sont pas développés" confirme les difficultés que les lexicographes rencontrent à définir l'enfance de façon ferme et univoque. Elle est définie par défaut, comme un manque. L'enfant n'est pas un adulte et il est appréhendé par rapport à l'adulte. Il n'est même utile de s'attarder sur les difficultés que soulève le sens de « développés ». Que signifie cet adjectif?

La définition de l'enfance n'est pas donnée, mais construite. Aux enfants sont attribuées en propre des caractéristiques qui les définissent, physiques, morales ou sociales, et qui varient suivant les dictionnaires. Les traits physiques ou physiologiques sont, dans le Trésor de la Langue Française, "les premières années de la vie et la puberté»; dans l' Universalis, "la locomotion, la marche, l'apprentissage du langage ; chez Chemin, «la faiblesse physique»; dans le Dictionnaire Général de la Langue Française, "le corps qui n'est pas encore développé ». Les traits moraux sont "l'absence de maturité ou la puérilité», de sorte que le nom enfance, au figuré, signifie, selon Littré, «l'état de puérilité prolongé dans le reste de la vie"; selon les auteurs du Trésor de la Langue Française, un enfant est aussi, par analogie, "un adulte qui offre, notamment dans son comportement physique, l'innocence, la fraîcheur d'un enfant » et "une personne adulte peu évoluée, immature ». Les traits sociaux définissant l'enfance relèvent de la filiation et de l'intégration dans un groupe ou dans une communauté. La filiation peut être naturelle: c'est la succession des êtres dans la durée. Elle pose le problème du renouvellement des générations et de la perpétuation de l'espèce. Elle peut être aussi spirituelle, les enfants étant les réceptacles de valeurs ou devant les assimiler pour les faire leurs. Aujourd'hui, la filiation est source de problèmes, du moins selon les rédacteurs de la revue Esprit (décembre 1996, "malaise dans la filiation »), à cause de la démographie (les générations se renouvellent difficilement) et de la transmission des valeurs, dont celles de la culture, qui se fait mal ou ne se fait plus ( $C f$., les thèses de Hannah Arendt et d'Alain Finkielkraut). ${ }^{8}$ Les autres traits sociaux se rapportent à l'intégration dans un groupe. L'enfant est d'abord membre d'un groupe, étroit (la famille ou l'école) ou large (la communauté ou la nation).

Ces hésitations, imprécisions, tâtonnements montrent que la définition de l'enfance dépend de l'idée que l'on se fait de l'homme. Est-ce un animal social ? Est-il caractérisé par des traits physiques ou physiologiques ou moraux? De fait, dans les dictionnaires et surtout dans les encyclopédies, apparaissent les enjeux idéologiques et moraux, dont l'enfance est le centre. 


\section{Autonomie ou subordination?}

Chemin définit l'enfance comme le "premier âge de la vie », auquel succèdent la jeunesse, à partir de 12 ou 13 ans, puis l'âge mûr, à partir de 21 ans, enfin la vieillesse qui commence lorsque les forces de l'âge mûr s'épuisent. Pour lui, l'essentiel est d'imposer aux enfants un système de valeurs, morales et spirituelles, afin qu'ils s'intègrent non seulement à une société politique mais aussi à une civilisation. De ce point de vue, bien que l'adjectif républicain du titre - qui annonce une rupture avec le monde latin - affirme explicitement le contraire, il n'y a pas de véritable coupure entre l'Ancien Régime et le nouveau, qui commence en 1789.

Deux siècles plus tard, dans l'Universalis, l'objectif essentiel se situe à l'exact opposé : on n'impose plus des valeurs, mais on laisse s'exprimer un désir (l'éducation ne doit pas plus être un obstacle) ou on laisse sourdre une parole. Dans cette encyclopédie, le point de vue à partir duquel est traitée la question de l'enfance est celui des sciences humaines et sociales (psychologie, sociologie, anthropologie et surtout psychanalyse), lesquelles, en dépit du terme sciences qui les désigne, ont un point de vue fortement préformé ou déformé par les présupposés idéologiques et moraux, comme le prouve l'analyse que développe, dans "les mythes de l'enfant dans notre société", $\mathrm{M}^{\mathrm{me}}$ Colette Misrahi, psychanalyste et partisan de l'antipsychiatrie. Selon cet auteur, l'enfant serait méconnu dans son être, parce qu'il est réduit à la représentation que l'adulte se fait de lui, c'est-àdire au rôle que l'adulte lui attribue dans la société, devenant le support ou le masque ou le refuge de la névrose de ses parents - proposition qui implique que tous les parents sont névrosés (!). Il est traité comme l'objet d'un savoir technocratique et, de lui, on attend rendement et efficacité. Les tests, de niveau ou le QI, le jaugent, l'évaluent, le classent. Pour en finir avec cette vision idéologique de l'enfant, $\mathrm{M}^{\mathrm{me}}$ Colette Mizrahi propose que l'on ne considère plus l'enfant comme une «nature » (ce qu'il serait pour les spécialistes), mais comme un sujet parlant et un sujet du désir, ce qu'il est. Pour le rendre à lui-même, il faut lui rendre une parole dont il est privé ou qu'il n'a jamais eue, comme le font les tenants de l'antipsychiatrie ou les disciples de Laing et de Cooper. Les adultes doivent prêter attention à la parole non dite, au désir non exprimé (c'est l'écoute psychanalytique). Quand la société et ses institutions seront remises en question, l'enfant pourra enfin être « libéré ».

Pour Chemin, ce qui caractérise l'enfance, c'est l'absence de toute autonomie, à quelque niveau qu'elle se situe : psychologique, matériel, moral, spirituel. Pour $\mathrm{M}^{\mathrm{me}}$ Mizrahi, c'est une autonomie de sujet parlant, désirant et agissant que les adultes doivent rendre à l'enfant, pour qu'enfin, il soit ou qu'il advienne.

\section{Une instruction publique?}

Ces présupposés idéologiques doivent nous amener à nous interroger sur le sens de l'adjectif républicain qui figure dans le titre de ce livret. Chemin apprend à lire aux enfants avec le même objectif que tous les idéologues, quelque régime totalitaire qu'ils servent, afin de les faire adhérer aux principes du nouveau régime, même s'ils ne les comprennent pas (en théorie, la liberté, l'égalité, les vertus républicaines,) et afin qu'ils entendent la Déclaration des droits de l'homme et la Constitution de 1793 ; en réalité, c'est 
une morale tirée d'a priori religieux qu'il impose autoritairement. Dans ce cadre, la lecture n'est pas une fin en soi, elle est instrumentalisée. Apprendre à lire, ce n'est pas se former, être soi, goûter aux plaisirs de la fable, c'est se voir imposer, sur le mode de la transmission autoritaire, une propagande. Or, à peu près au même moment, Condorcet établit les principes de l'instruction publique, qui inspireront Jules Ferry et les hommes politiques des années 1880 jusqu'en 1940. Pour Condorcet, qui défend la nécessité du suffrage universel, un peuple, en votant librement, peut choisir des tyrans et aliéner sa liberté. C'est pourquoi il faut l'instruire et établir, dans la République, une véritable instruction publique, qui ne soit pas de la propagande, mais qui fonde la liberté de l'esprit, en favorise le développement, et qui soit une vraie propédeutique à la liberté, en enseignant des éléments de connaissance, en apprenant aux futurs citoyens à observer, à mesurer, à peser, à calculer, à lire ET écrire en même temps. Il ne s'agit pas pour un maître d'informer ses élèves de l'existence d'une chose, mais de démontrer par le raisonnement que la chose est, ou que la loi physique s'applique dans tel ou tel phénomène. L'objectif final est qu'un maître ou magister apprenne à ses élèves à se passer de maitre ou dominus ; qu'il les arrache à ce qui les détermine ; en un mot, il s'agit de les émanciper (comme la loi émancipe les esclaves en les libérant). Ces objectifs et ces principes se situent à l'opposé exact de ceux qui sous-tendent la méthode de Chemin, qui n'a rien de républicain, en dépit de ce que proclame le titre de son Alphabet [...]. Il faudra environ un siècle, et attendre 1882, pour que les objectifs de l'apprentissage de la lecture ne se ramènent plus nécessairement - du moins dans les principes - à l'imposition d'une propagande explicite et militante.

\section{BIBLIOGRAPHIE}

BEACCO J.-C. : « Approche communicative : quels rythmes méthodologiques ? », in Le Français dans le Monde, $\mathrm{N}^{\circ}$ 248, avril 1992. Paris, Éd. conjointe : Hachette - Larousse.

BOUTON C.P. : Les grammaires de Claude Mauger. Paris, Klincksieck, 1971.

COSTE D. : « Les discours naturels de la classe », in Le Français dans le Monde, $\mathrm{N}^{\circ} 183$, févriermars 1984. Paris, Éd. conjointe : Hachette-Larousse.

CHEVALIER J.-C. : La notion de complément chez les grammairiens. Genève, Droz, 1968.

GANASCIA J.G. : Les sciences cognitives. Paris, Flammarion, Collection : Dominos, 1996.

GAYET D. : Modèles éducatifs et relations pédagogiques. Paris, A. Colin, 1995.

GAONAC'H : Psychologie cognitive et éducation, implications dans l'enseignement des langues

étrangères, Thèse pour le doctorat en psychologie. Dijon, Éd. : Université de Dijon, 1986.

HYMES D. : Vers la compétence de communication. Paris, Éd. conjointe : Crédif - Hatier, Collection : LAL, 1991.

PERRAUDEAU M. : Les méthodes cognitives, Apprendre autrement à l'école. Paris,Armand Colin, 1996. 
PUREN C. : La didactique des langues étrangères à la croisée des méthodes - Essai sur l'éclectisme. Paris, Didier, 1994.

PUREN C. : Histoire des méthodologies. Paris, Didier, 1989.

\section{NOTES}

1. ARCHIVES NATIONALES, Musée de l'Histoire de France (reproduit en fac-similé). Paris, Editions de la Réunion des Musées Nationaux et Archives nationales, 1990.

2. Cf., l'ouvrage collectif - Les Préaux de la République. Minerve, 1993.

3. Cf., ses principaux textes recueillis dans - L'école ou le loisir de penser. Editions du CNDP 1994.

4. Cf., Dictionnaire biographique. 1973.

5. CERTEAU M. De : Une politique de la langue. Paris, Gallimard, 1994.

6. Reproduit dans - GAULUPEAU Y.: La France à l'école. Paris, Gallimard, Collection: Découvertes, 1992.

7. Ces textes de loi sont reproduits partiellement dans La France à l'école,. Op. Cit.

8. ARENDT H. : Crise de la culture. Paris, Folio. FINKIELKRAUT A. : La Défaite de la pensée. Paris, Gallimard.

\section{AUTEUR}

\section{JEAN-GÉRARD LAPACHERIE}

Université de Pau 